\title{
Research Note \\ PSR B1929+10 and GSC 01060-01374 are not binary
companions ${ }^{\star}$
}

\author{
M. L. A. Kouwenhoven and M. C. van den Berg \\ Astronomical Institute, PO Box 80000, 3508 TA Utrecht, The Netherlands \\ Received 7 September 2000 / Accepted 23 October 2000

\begin{abstract}
We have observed the star GSC 01060-01374 to investigate whether it is in a binary with PSR B1929+10. Its spectral type is K4-6 and its luminosity class is III or II, therefore its distance is $2.4 \mathrm{kpc}$ or higher. Since the dispersion measure distance of PSR B1929+10 is $0.17 \mathrm{kpc}$, we rule out the possibility that these two stars are associated in a binary. This poses further constraints on the lower limit of kick velocities in supernova explosions.
\end{abstract}

Key words. stars: individual: GSC 01060-01374 - stars: neutron - pulsars: individual: PSR B1929+10

\section{Introduction}

Portegies Zwart et al. (1997) have compared the positions of pulsars from the pulsar catalog (Taylor et al. 1993, 1995) with the positions of stars in the Guide Star Catalog (GSC, Lasker et al. 1990; Jenker et al. 1990). They noticed that the position of the pulsar PSR B1929+10 and that of the star GSC 01060-01374 differ by only $4.87^{\prime \prime}$, which corresponds to a separation of $0.004 \mathrm{pc}$ at the distance of the pulsar. PSR B1929+10 is a nearby radio pulsar with a period of $0.227 \mathrm{~s}$ and a spin-down age of $310^{6}$ yr (Large et al. 1968). Its dispersion measure is only $3.176 \pm 0.003 \mathrm{~cm}^{-3} \mathrm{pc}$, which leads to a distance estimate of $0.17 \mathrm{kpc}$. Distances obtained from dispersion measures are believed to be accurate to $\sim 25 \%$ (Taylor \& Cordes 1993). This result is in agreement with the parallax distance of $0.20 \pm 0.06 \mathrm{kpc}$ found by Campbell (1995). However, Backer \& Sramek (1982) found a slightly higher lower limit to the parallax distance of $0.25 \mathrm{kpc}$.

Portegies Zwart et al. (1997) showed that one known radio pulsar is expected to be in a wide binary with a star in the GSC, if kicks in supernova explosions exist and have a velocity of about $10 \mathrm{~km} \mathrm{~s}^{-1}$. For higher kick velocities

Send offprint requests to: M. L. A. Kouwenhoven, e-mail: M.L.A.Kouwenhoven@astro.uu.nl

* Based on observations made with the William Herschel Telescope operated on the island of La Palma by the Isaac Newton Group in the Spanish Observatorio del Roque de los Muchachos of the Instituto de Astrofisica de Canarias. no correlation between stars from the pulsar catalog and the GSC is expected, since these kicks will then dissociate all wide binaries. Therefore, if PSR B1929+10 is indeed in a binary, small kick velocities must exist and the kick velocity distribution must have a low-velocity tail. In this note we investigate the possible binary relation between PSR B1929+10 and GSC 01060-01374.

\section{Observation and data reduction}

We have taken a low-resolution spectrum $(R \approx 1000)$ of the star GSC $01060-01374$ on July 31, 1999 with the ISIS dual-beam spectrograph mounted on the $4.2 \mathrm{~m}$ William Herschel Telesope on La Palma.

We observed the star for $180 \mathrm{~s}$ in both the blue (2743-6260 $\AA$ ) and red (5778-8727 $\AA$ ) arm of ISIS with the spectrograph slit width set to $4^{\prime \prime}$. The flux standard $\mathrm{BD}+284211$ was observed for $30 \mathrm{~s}$ in both arms, with the slit widened to $8^{\prime \prime}$. The seeing during these observations was between 0.8 and $11^{\prime \prime} 1$.

After removal of the bias signal, extraction of the spectrum and flat-fielding, the spectrum was converted to an absolute flux scale. For that purpose we used the observation of the flux standard, its standard spectrum (Massey et al. 1988) and an atmospheric extinction curve for La Palma (King 1985). Spectra of CuNe-lamps were used to do the wavelength calibration. The spectrum is displayed in Fig. 1. The flux calibration between 5700 and $5850 \AA$ was not reliable, as the response of the CCDs in the 


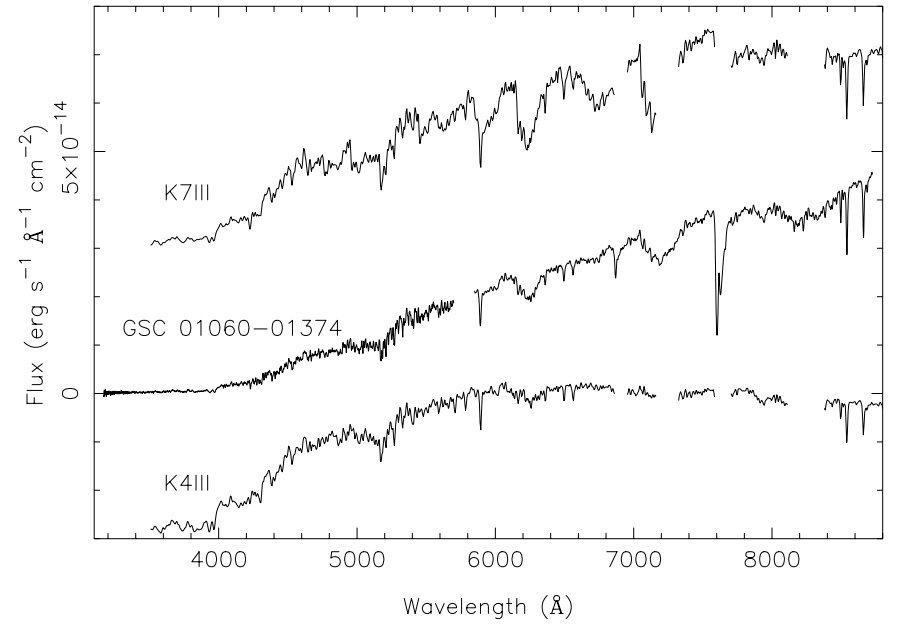

Fig. 1. Observed spectrum of the star GSC 01060-01374 and scaled and shifted spectra of standard K4III and K7III stars from Silva \& Cornell (1992). The difference in the slope between the spectra of our star and that of the standard stars is probably due to reddening

dispersion direction is highly variable in that region. For more details about the observations and data reduction we refer to van den Berg \& Verbunt (in preparation).

\section{Spectral classification}

We derive that the luminosity class of GSC 01060-01374 is I, II or III from the classification criteria for late $\mathrm{K}$ to $\mathrm{M}$ stars in Figs. 6d and 7d in Kirkpatrick et al. (1991) and in particular from the strength of the Ca II line at $8542 \AA$ relative to the nearby continuum. We find a ratio $D$ (see Kirkpatrick et al. 1991) of $1.36 \pm 0.02$ and ratio $D /$ ratio $C$ of $1.31 \pm 0.03$. We can not distinguish between luminosity classes I, II and III, since the number of comparison stars is limited.

The spectral type K4-6 is derived from the index $I_{\mathrm{S}}$ as defined in Malyuto \& Schmidt-Kaler (1997) that includes features between $4215-4360 \AA$ and $5125-5290 \AA$. We find an index $I_{\mathrm{S}}=0.274 \pm 0.015$. The indices $I_{2}$ and $I_{3}$, also taken from Malyuto \& Schmidt-Kaler, suggest a luminosity class III-I as well. We conclude that GSC 01060-01374 is a $\mathrm{K}(4-6)$ III-I star.

Transmission curves of $U, B$ and $V$ filters are used to obtain $U, B$ and $V$ magnitudes from the flux calibrated spectra. We find $U=17.7 \pm 0.1, B=15.4 \pm 0.1$ and $V=13.4 \pm 0.1$. The errors are estimated by comparing the $B$ and $V$ magnitudes thus obtained for the flux standard with their tabulated values. The observed apparent $V$ magnitude is in accordance with the value of $12.9 \pm 0.4$ from the GSC.

The observed colours are in agreement with our spectral classification. If our star has luminosity class III the difference in the observed and expected $B-V$ is $0.6 \pm 0.2$. From this we derive that the reddening in $V$ is: $A_{V}=1.7 \pm 0.6$. If our star has luminosity class II or I, $A_{V}=1.9 \pm 0.4$ or $A_{V}=1.6 \pm 0.4$, respectively.

Since the absolute $V$ magnitude $M_{V}$ of a class III, II and I K5 star are $-0.2,-2.3$ and -5.8 , respectively, we can now derive the distance $D$ to the star using the relation:

$V-M_{V}=5 \log D-5+A_{V}$.

This distance equals $2.4 \pm 0.8,5.8 \pm 1.2$ and $33 \pm 7 \mathrm{kpc}$ for a class III, II and I star, respectively. The galactic longitude of the star is $47^{\circ}$ and latitude is $-4^{\circ}$. We consider it very unlikely that a supergiant is located at a distance of $33 \mathrm{kpc}$ in this direction. We therefore conclude that the star is a giant or a bright giant and its distance is $2.4 \mathrm{kpc}$ or higher. This distance is also in good agreement with the observed reddening.

\section{Conclusion}

Since the distance to the pulsar is only $0.17 \mathrm{kpc}$, we exclude the possibility of a binary association between PSR B1929+10 and GSC 01060-01374, even if we take the large uncertainties of the dispersion-distance relation for nearby pulsars into account. We find no evidence for a low velocity tail in the kick velocity distribution and the average kick velocity should be at least $10 \mathrm{~km} \mathrm{~s}^{-1}$ (Portegies Zwart et al. 1997).

Acknowledgements. MK is supported by The Netherlands Research School for Astronomy (NOVA), a national association of astronomy departments at the Universities of Amsterdam, Groningen, Leiden and Utrecht. MvdB is supported by the Netherlands Organization for Scientific Research (NWO).

\section{References}

Backer, D. C., \& Sramek, R. A. 1982, ApJ, 260, 512

Campbell, R. M. 1995, Ph.D. Thesis, Harvard University

Jenkner, H., Lasker, B. M., Sturch, C. R., et al. 1990, AJ, 99, 2081

King, D. L. 1985, ING Technical Note 31

Kirkpatrick, J. D., Henry, T. J., \& McCarthy Jr., D. W. 1991, ApJS, 77, 417

Large, M. I., Vaughan, A. E., \& Wielebinski, R. 1968, Nature, 220,753

Lasker, B. M., Sturch, C. R., McLean, B. J., et al. 1990, AJ, 99, 2019

Malyuto, V., \& Schmidt-Kaler, T. 1997, A\&A, 325, 693

Massey, P., Strobel, K., Barnes, J. V., \& Anderson, E. 1988, ApJ, 328, 315

Portegies Zwart, S. F., Kouwenhoven, M. L. A., \& Reynolds, A. P. 1997, A\&A, 328, L33

Silva, D. R., \& Cornell, M. E. 1992, ApJS, 81, 865

Taylor, J. H., \& Cordes, J. M. 1993, ApJ, 411, 674

Taylor, J. H., Manchester, R. N., \& Lyne, A. G. 1993, ApJS, 88,529

Taylor, J. H., Manchester, R. N., Lyne, A. G., \& Camilo, F. 1995, Catalog of 706 Pulsars, Unpublished work, available via http://pulsar.princeton.edu/pulsar/ catalog.shtml 